\title{
THE CRITICAL POINTS OF LINEAR COMBINATIONS OF HARMONIC FUNCTIONS
}

\author{
J. L. WALSH
}

In various extremal problems of function theory the critical points of linear combinations of Green's functions and harmonic measures are of significance. ${ }^{1}$ The object of the present note is to indicate some of the more immediate results concerning the location of such critical points, for both simply and multiply connected regions.

The first configuration to be studied is a simple one. In the $z$-plane let $C$ be the unit circle and let $\alpha_{1}$ be an arc of $C$, whose initial and terminal points are $a_{1}$ and $b_{1}$ respectively, the positive direction chosen as counterclockwise. Let $\omega\left(z, \alpha_{1}, R\right)$ denote generically the harmonic measure of $\alpha_{1}$ in the point $z$ of $R$ with respect to the region $R$; that is to say, $\omega$ is the function which is harmonic and bounded in $R$, continuous in the corresponding closed region except in the end points of $\alpha_{1}$, equal to unity in the interior points of $\alpha_{1}$ and to zero in the interior points of the boundary arcs complementary to $\alpha_{1}$. The reader will verify the equation

$$
\omega\left(z, \alpha_{1},|z|<1\right)=\frac{1}{\pi}\left[\arg \left(z-b_{1}\right)-\arg \left(z-a_{1}\right)-\frac{1}{2} \alpha_{1}\right] .
$$

If $\zeta$ is a point interior to $C:|\zeta|<1$, Green's function for $R:|z|<1$ with pole in the point $\zeta$ can be written

$$
g(z, \zeta, R)=\log |1-\bar{\zeta} z|-\log |z-\zeta| .
$$

An arbitrary linear combination of $g$ and $\omega$ with real constant coefficients is $U(z)=\lambda g+\mu \omega$, which is the real part of the analytic function

$$
f(z)=\lambda \log \frac{1-\bar{\zeta} z}{z-\zeta}+\frac{\mu}{i \pi}\left[\log \left(z-b_{1}\right)-\log \left(z-a_{1}\right)-\frac{i \alpha_{1}}{2}\right] .
$$

The critical points of $U(z)$ are precisely the critical points of $f(z)$, namely the zeros of

$$
i f^{\prime}(z)=\frac{i \lambda}{z-1 / \bar{\zeta}}-\frac{i \lambda}{z-\zeta}+\frac{\mu}{\pi}\left[\frac{1}{z-b_{1}}-\frac{1}{z-a_{1}}\right] .
$$

Presented to the Society, September 4, 1947; received by the editors June 16, 1947.

${ }^{1}$ See, for instance, M. Schiffer, Amer. J. Math. vol. 68 (1946) pp. 417-448; L. V. Ahlfors, Duke Math. J. vol. 14 (1947) pp. 1-11. 
We study the zeros of $f^{\prime}(z)$ by setting up a field of force suggested by the individual terms in the second member of (3); it is more convenient to replace each term by its conjugate. If particles are chosen to repel with a force equal to the mass divided by the distance, the force at $z$ due to a positive unit particle at $b_{1}$ is $1 /\left(\bar{z}-\bar{b}_{1}\right)$; the force at $z$ due to particles at $a_{1}$ and $b_{1}$ of respective masses $-\mu / \pi$ and $+\mu / \pi$ is the conjugate of the last term of (3). We introduce also skew particles, of such a nature that a skew particle at $z_{0}$ exerts a force at $z$ equal to the mass divided by the distance, in the direction arg $\left(z-z_{0}\right)$ $+\pi / 2$ for positive mass and in the reverse direction for negative mass. ${ }^{2}$ The force at $z$ due to skew particles of respective masses $\lambda$ and $-\lambda$ at the points $\zeta$ and $1 / \xi$ is the conjugate of the sum of the first two terms of (3); the critical points of $U(z)$ are then precisely the positions of equilibrium in the field of force due to the set of four particles.

The function $U(z)$ is invariant under conformal transformation of the interior of $C$ into itself; we study for convenience the origin $O$ as a possible critical point. The force at $O$ due to the particles at $b_{1}$ and $a_{1}$ is $\mu / \pi$ times the sum of the vectors $b_{1} O$ and $O a_{1}$, which equals $\mu / \pi$ times the vector $b_{1} a_{1}$. The force at $O$ due to the skew particles at $\zeta$ and $1 / \bar{\zeta}$ is in magnitude $\lambda$ times the difference $(1 / \zeta)-\zeta$, and if $\lambda>0$ is exerted in the direction $-\arg \zeta+\pi / 2$. The point $O$ can be a position of equilibrium only if these two forces act in opposite directions, so if $\lambda \mu<0$, the point $\zeta$ lies between $O$ and $\alpha_{1}$ on the radius bisecting the arc $\alpha_{1}$; if $\lambda \mu>0$, the point $O$ can be a position of equilibrium only if $\zeta$ lies on this same diameter but is separated by $O$ from $\alpha_{1}$. An easy topological discussion, or a further study of the field of force, shows that $U(z)$ has at most a unique critical point interior to $C$. We express this entire result no longer requiring the position of equilibrium to lie at the center of $C$; moreover the result applies to an arbitrary Jordan region $R$, with non-euclidean geometry defined in $R$ by mapping $R$ onto the interior of the unit circle:

TheOREM 1. If $\alpha_{1}$ is an arc of the Jordan curve $C$ whose interior is $R$, and if $\zeta$ is an arbitrary point of $R$, then for $\lambda \mu<0$ the unique critical point if any of $U(z)=\lambda g(z, \zeta, R)+\mu \omega\left(z, \alpha_{1}, R\right)$ in $R$ lies on the noneuclidean line through $\zeta$ bisecting $\alpha_{1}$ (in the sense that the angles subtended at $\zeta$ by the two parts of $\alpha_{1}$ are equal), but does not lie between $\zeta$ and $\alpha_{1}$; for $\lambda \mu>0$, the unique critical point of $U(z)$ if any in $R$ lies on this same non-euclidean line between $\zeta$ and $\alpha_{1}$.

${ }^{2}$ If a right forearm, thumb away from the plane, points from $z_{0}$ to $z$, then the sense of the force exerted at $z$ by a positive skew particle at $z_{0}$ is indicated by the fingers at right angles to the forearm. Thus a positive skew particle can be considered as a right skew particle, and similarly a negative skew particle as a left skew particle. 
We now generalize Theorem 1 so as to permit any finite number of Green's functions and harmonic measures:

TheORem 2. Let $R$ be a region bounded by a Jordan curve $C$, let $\zeta_{1}, \zeta_{2}, \cdots, \zeta_{m}$ be points of $R$ and $\alpha_{1}, \alpha_{2}, \cdots, \alpha_{n}$ be arcs of $C$. We form the linear combination with real constant coefficients

$$
U(z)=\sum_{k=1}^{m} \lambda_{k} g\left(z, \zeta_{k}, R\right)+\sum_{k=1}^{n} \mu_{k} \omega\left(z, \alpha_{k}, R\right) .
$$

Let $\lambda_{1}, \lambda_{2}, \cdots, \lambda_{m^{\prime}}$ and $\mu_{1}, \mu_{2}, \cdots, \mu_{n^{\prime}}$ be positive, and the remaining $\lambda_{k}$ and $\mu_{k}$ be negative.

All non-euclidean lines $L$ (if any) for $R$ each of which separates the points $\zeta_{1}, \zeta_{2}, \cdots, \zeta_{m^{\prime}}$ and the arcs $\alpha_{1}, \alpha_{2}, \cdots, \alpha_{n^{\prime}}$ from the points $\zeta_{m^{\prime}+1}, \cdots, \zeta_{m}$ and the arcs $\alpha_{n^{\prime}+1}, \cdots, \alpha_{n}$ lie in a locus $\Pi$ which is a subregion of $R$ in which no critical points of $U(z)$ lie. Each such line $L$ separates a subset $R_{1}$ of $R+C$ from a subset $R_{2}$ of $R+C$, where $R_{1}$ is non-euclidean convex and contains the points $\zeta_{k}\left(k=1,2, \cdots, m^{\prime}\right)$ and the arcs $\alpha_{k}\left(k=1,2, \cdots, n^{\prime}\right)$, and $R_{2}$ is non-euclidean convex, disjoint from $R_{1}$, and contains all the remaining points $\zeta_{k}$ and arcs $\alpha_{k}$. If the non-euclidean line $L$ separates all the points $\zeta_{k}\left(k=1,2, \cdots, m^{\prime}\right)$ not on $L$ and all the interior points of the arcs $\alpha_{k}\left(k=1,2, \cdots, n^{\prime}\right)$ from all the remaining points $\zeta_{k}$ not on $L$ and all the interior points of the remaining arcs $\alpha_{k}$, then no point of $L$ in $R$ is a critical point of $U(z)$ unless all $\zeta_{k}$ lie on $L$ and $n$ is zero.

In Theorem 2 the $\operatorname{arcs} \alpha_{k}$ may overlap and may have end points in common.

In the proof of Theorem 2 , let $R$ be the interior of the unit circle, let $L$ be the vertical diameter, let the points $\zeta_{k}\left(k=1,2, \cdots, m^{\prime}\right)$ not on $L$ and the $\operatorname{arcs} \alpha_{k}\left(k=1,2, \cdots, n^{\prime}\right)$ except perhaps end points lie to the right of $L$; the remaining $\zeta_{k}$ not on $L$ and the remaining arcs $\alpha_{k}$ except perhaps end points lie to the left of $L$. We consider the point $z=0$ as a possible position of equilibrium in the field of force corresponding to that used in proving Theorem 1 , where we now combine the fields for all $m$ Green's functions and for all $n$ harmonic measures involved; the positions of equilibrium in $R$ are precisely the critical points of $U(z)$ in $R$. The force at $O$ due to each pair of particles at end points of an $\operatorname{arc} \alpha_{k}\left(k=1,2, \cdots, m^{\prime}\right)$ has a nonvanishing component directed vertically downward, as has the force at $O$ due to each pair of particles at end points of an arc $\alpha_{k}\left(k=n^{\prime}+1, n^{\prime}+2, \cdots, n\right)$. Likewise the force at $O$ due to each pair of skew particles at points $\zeta_{k}$ and $1 / \zeta_{k}$ not on $L$ has a nonvanishing component directed vertically downward, whether $k \leqq m^{\prime}$ or $k>m^{\prime}$; for pairs of skew particles on $L$, 
the force exerted at $O$ is horizontal. Thus the total force at $O$ cannot be zero, and $O$ cannot be a position of equilibrium nor a critical point of $U(z)$. This completes the proof of Theorem 2 except for fairly obvious geometric relationships which are left to the reader.

In Theorem 2 the case $m^{\prime}=m, n^{\prime}=n$ is not excluded:

Corollary 1. Under the conditions of Theorem 2, suppose $m^{\prime}=m$, $n^{\prime}=n$. If $\Pi$ is a non-euclidean convex closed region in the closure of $R$ which contains all the $\zeta_{k}$ and $\alpha_{k}$, then $\Pi$ contains also all critical points of $U(z)$ in $R$. More explicitly, if a non-euclidean line $L$ passes through a point $z$ of $R$, if all the points $\zeta_{k}$ not on $L$ lie on one side of $L$, and if all the arcs $\alpha_{k}$ except perhaps end points lie on this same side of $L$, where if all $\zeta_{k}$ lie on $L$ we assume $n>0$, then $z$ is not a critical point of $U(z)$.

In the case under Corollary 1 with $\mu_{1}=\mu_{2}=\cdots=\mu_{n}=1$ and the $\alpha_{k}$ disjoint, a non-euclidean convex region $\Pi_{1}$ can be defined in $R+C$ containing the arcs $\alpha_{k}$ (loci $U(z)=1$ ) and also the loci $U(z)=1$ interior to $R$; one of the latter loci must clearly separate each $\zeta_{k}$ from $C$. It is then known ${ }^{3}$ that $\Pi_{1}$ contains all critical points of $U(z)$ in $R$; but the minimal $\Pi_{1}$ always contains the minimal region $\Pi$, and may be larger than $\Pi$, so Corollary 1 is sharper in this particular situation than the previous result.

Likewise in the more general situation of Theorem 2 itself, with $\left|\mu_{1}\right|=\left|\mu_{2}\right|=\cdots=\left|\mu_{n}\right|=1$ and the $\alpha_{k}$ disjoint, a result similar to Theorem 2 is known (loc. cit.) involving the arcs $\alpha_{k}$ (loci $U(z)= \pm 1$ ) and the loci $U(z)= \pm 1$ interior to $R$; one of the latter separates each $\zeta_{k}$ from $C$. Theorem 2 is again sharper in this particular situation than the previous result.

In certain cases, Theorem 2 itself can be somewhat improved:

Corollary 2. In Theorem 2 with $m^{\prime}=m, n^{\prime}=n=1$, no critical point of $U(z)$ lies on a non-euclidean line $L$ which cuts $\alpha_{1}$ but separates all the $\zeta_{k}$ from the end points of $\alpha_{1}$.

In Theorem 2 with $m^{\prime}=0, n^{\prime}=n=1$, any subregion of $R$ bounded by a subarc $\alpha$ of $\alpha_{1}$ and by a non-euclidean line joining the end points of $\alpha$, and which contains no point $\zeta_{k}$, is free from critical points of $U(z)$.

Corollary 2 is intended to be suggestive rather than exhaustive of the method. The proof, which is left to the reader, follows closely the proof of Theorem 2.

A new interpretation of Corollary 2 is found by denoting by $\beta_{1}$ the arc of $C$ complementary to $\alpha_{1}$, and by setting $\omega\left(z, \alpha_{1}, R\right)=1-\omega\left(z, \beta_{1}, R\right)$. The critical points of $U(z)$ are the same as those of the function

${ }^{3}$ Walsh, Bull. Amer. Math. Soc. vol. 54 (1948) pp. 191-195. 


$$
U(z)-\mu_{1}=\sum_{k=1}^{m} \lambda_{k} g\left(z, \zeta_{k}, R\right)-\mu_{1} \omega\left(z, \beta_{1}, R\right), \quad \mu_{1}>0,
$$

to which the application of Theorem 2 yields Corollary 2 .

In comparison with all previous existing theorems, Theorem 2 is especially novel in that it applies to harmonic functions which assume in addition to zero more than two distinct values on prescribed point sets.

If a nonconstant harmonic function $U(z)$ is the uniform limit of a sequence of harmonic functions $U_{p}(z)$, the critical points of $U(z)$ are precisely the limits of the critical points of the $U_{p}(z)$. It is thus clear that Theorem 2 applies under suitable conditions when the two sums in (4) are replaced by the limits of similar sums. If for each function of a uniformly convergent sequence $U_{p}(z)$ the set $R_{1}$ of Theorem 2 contains all the points $\zeta_{k}$ and all the arcs $\alpha_{k}$ corresponding to positive coefficients $\lambda_{k}$ and $\mu_{k}$, and if the set $R_{2}$ contains all the points $\zeta_{k}$ and all the arcs corresponding to negative coefficients $\lambda_{k}$ and $\mu_{k}$, then the region $\Pi$ if independent of $p$ contains no critical point of the limit (assumed nonconstant) of the sequence. We proceed to apply this remark, by proving the following corollary.

Corollary 3. Let $R$ be a Jordan region bounded by a curve $C$, let the function $U_{1}(z)$ be bounded and continuous on $C$ except perhaps for a finite number of discontinuities, and let $U(z)$ be harmonic and bounded in $R$, and continuous and equal to $U_{1}(z)$ at every point of continuity of $U_{1}(z)$ on $C$. Let all points of $C$ at which $U_{1}(z)$ is positive be contained in an arc $\alpha$ of $C$, and all points of $C$ at which $U_{1}(z)$ is negative be contained in an arc $\beta$ of $C$ disjoint from $\alpha$. Then all critical points of $U(z)$ (assumed not identically zero) in $R$ lie in the sets $\omega(z, \alpha, R) \geqq 1 / 2$, $\omega(z, \beta, R) \geqq 1 / 2$.

Corollary 3 is an immediate consequence of the representation

$$
U(z)=\int_{0}^{1} U_{1}(t) d \omega(z, t, R)
$$

and of the corresponding expression for $U(z)$ as the limit of a sum. If the $\operatorname{arcs} \alpha$ and $\beta$ are minimal, the locus $\Pi$ is bounded by the arcs $\omega(z, \alpha, R)=1 / 2, \omega(z, \beta, R)=1 / 2$.

An easy immediate proof of Corollary 3 can be given by the usual form of Poisson's integral for the unit circle $C$; it is again convenient to choose the origin as the point studied, with the axis of imaginaries separating the intervals of $C$ on which $U_{1}(z)$ is positive from the intervals of $C$ on which $U_{1}(z)$ is negative; assume the latter intervals 
to lie to the left of that axis. Direct computation shows the partial derivative of $U(z)$ at the origin in the positive horizontal direction to be positive:

$$
U^{\prime}(x)=\frac{1}{\pi} \int_{0}^{2 \pi} U_{1} \cos \theta d \theta>0,
$$

so the origin is not a critical point of $U(z)$. This new proof (valid also in three dimensions) has the advantage over the previous one of slightly generalizing Corollary 3 by not requiring $U_{1}(z)$ to vanish on an entire arc of $C$. Theorem 2 itself can be proved by a similar procedure. Nevertheless the original proof of Theorem 2 is to be considered as intuitively suggestive and useful, as applying at once in the important case of linear combinations of harmonic measures, and as exhibiting relations with other proofs on the location of critical points.

Essentially a special case of Corollary 3 occurs if $U_{1}(z)$ is non-negative on $C$ :

Corollary 4 . Let $R$ be a Jordan region bounded by a curve $C$, let the function $U_{1}(z)$ be bounded, non-negative, and continuous on $C$ except perhaps for a finite number of discontinuities, and let $U(z)$ be harmonic and bounded in $R$, continuous and equal to $U_{1}(z)$ at every point of continuity of $U_{1}(z)$ on $C$. Then any non-euclidean convex region of $R+C$ which contains all points of $C$ at which $U_{1}(z)$ is positive contains also all critical points of $U(z)$ in $R$. Thus if $U_{1}(z)$ vanishes on an arc $\beta$ of $C$, the region $\omega(z, \beta, R)>1 / 2$ contains no critical points of $U(z)$.

Just as the second sum of (4) can be replaced by the integral in (5), namely the limit of such a sum, so the first sum in (4) may be replaced by the sum of any number of functions of a certain class $K$, namely the class of functions each the uniform limit in a suitable subregion of $R$ of sums of the type $\sum_{k=1}^{m} \lambda_{k} g\left(z, \zeta_{k}, R\right)$. To the class $K$ belong: (a) Green's functions $g\left(z, \zeta_{k}, R\right)$; (b) any function $U(z)$ harmonic in a closed multiply connected subregion $R_{1}$ of $R$ bounded by $C$ and by a set of disjoint analytic Jordan curves $\Gamma$ interior to $R,{ }^{4}$ equal to zero on $C$ and to unity on $\Gamma$. Thus if $\nu$ denotes the exterior normal for $R_{1}$, we have

$$
U(z)=\frac{1}{2 \pi} \int_{\Gamma}\left(g \frac{\partial U}{\partial \nu}-U \frac{\partial g}{\partial \nu}\right) d s+\frac{1}{2 \pi} \int_{C}\left(g \frac{\partial U}{\partial \nu}-U \frac{\partial g}{\partial \nu}\right) d s
$$

which in the present case reduces to

\footnotetext{
${ }^{4}$ The requirement that $\Gamma$ be interior to $R$ can be weakened (loc. cit.).
} 
$U(z)=\frac{1}{2 \pi} \int_{\Gamma} g \frac{\partial U}{\partial \nu} d s$, $\frac{\partial U}{\partial \nu}>0$

obviously of class $K$. More generally, there belongs to class $K$ any function which can be expressed

$$
U(z)=\int_{\Gamma} g(z, t, R) V(t) d s_{t}
$$

where the function $V(t)$ is integrable on $\Gamma$. Theorem 2 thus extends to apply to any sum of a function of class $K$ and a function of type (5).

In this extension of Theorem 2, proper attention must of course be given to the signs of the functions $U_{1}(z)$ in (5) and $V(t)$ in (6), and the question of the latter seems not to be easy. We note explicitly that not every function $U(z)$ harmonic and positive in $R_{1}$, assuming continuous values zero on $C$ and positive on $\Gamma$, admits a representation (6) where $V(t)$ is non-negative on $\Gamma$. We establish this result by display of a counter example. Let $R_{2}$ be the annular region bounded by the circles $C:|z|=1$ and $C_{2}:|z|=r^{2}<1$, which are mutually inverse in the circle $C_{1}:|z|=r$. Let Green's function for $R_{2}$ be $g\left(z, \zeta, R_{2}\right)$; we consider the function $U_{2}(z)=g\left(z, r, R_{2}\right)+g\left(z,-r, R_{2}\right)$, which has (as study of the level curves indicates) precisely two critical points $z_{1}$ and $z_{2}$ interior to $R_{2}$; this set of two points is symmetric in $C_{1}$ and in the axis of reals, and we set $z_{1}=i r, z_{2}=-i$. A suitably chosen circle $\gamma$ orthogonal to $C$, having its center on the axis of imaginaries, contains in its interior $z_{1}$ but no point of $C_{2}$. Let $\Gamma$ denote the sum of $C_{2}$ and the two level curves $\Gamma_{1}: U_{2}(z)=N(>0)$, where $N$ is chosen so large that $\Gamma_{1}$ is exterior to $\gamma$. The function $V_{p}(z)$ harmonic and continuous in the region $R_{1}$ bounded by $C$ and $\Gamma$, equal to zero on $C$, to $N$ on $\Gamma_{1}$, to $1 / p$ on $C_{2}$, approaches uniformly in $R_{1}$ as $p$ becomes infinite the limit $U_{2}(z)$. Moreover $V_{p}(z)$ is positive in $R_{1}$ and also on $\Gamma$. The critical points of $U_{2}(z)$ in $R_{1}$ are the limits of those of $V_{p}(z)$ in $R_{1}$. If we assume $V_{p}(z)$ to admit a representation of form

$$
V_{p}(z)=\int_{\Gamma} g(t, z, R) W_{p}(t) d s,
$$

where $W_{p}(t)$ is non-negative on $\Gamma$, it follows that $\gamma$, containing no point of $\Gamma$, contains no critical point of $V_{p}(z)$ and contains no critical point of $U_{2}(z)$, contrary to construction..$^{5}$

${ }^{5} \mathrm{~A}$ contradiction can be obtained here also by considering the integral of the normal derivative of $V_{p}(z)$ over a curve in $R_{1}$ for which $C_{2}$ is interior but $\Gamma_{1}$ is exterior. 
Theorem 2 and the suggested generalizations apply primarily to a simply connected region $R$, but if we start with a multiply connected region, functions harmonic there may be studied by mapping a covering surface onto a simply connected region. In preparation for this study we prove the following theorem.

THEOREM 3. Let the function $V(z)$ be harmonic in the upper halfplane $R$, except that in the neighborhood of each point $\rho^{n} \alpha$ ( $\alpha$ in $R$, $\rho>1, n=\cdots,-2,-1,0,1,2, \cdots)$ the sum $V(z)+\log \left|z-\rho^{n} \alpha\right|$ is harmonic. Let $V(z)$ be continuous and equal to zero at each finite point of the axis of reals other than $z=0$, and satisfy the functional equation $V(\rho z) \equiv V(z)$. Then we have the representation

$$
V(z)=\sum_{-\infty}^{\infty} \log \left|\frac{z-\rho^{n} \bar{\alpha}}{z-\rho^{n} \alpha}\right|,
$$

a series which converges uniformly in any closed bounded region of the plane not containing a point $\rho^{n} \alpha$ or the origin.

Each term of the series in (7) is Green's function for $R$ with pole in a point $\rho^{n} \alpha$. The function $V(z)$ is closely related to analytic functions introduced by Pincherle.

As $n$ becomes positively infinite we write

$$
\log \left|\frac{z-\rho^{n} \bar{\alpha}}{z-\rho^{n} \alpha}\right|=\log \left|\frac{1-z / \rho^{n} \bar{\alpha}}{1-z / \rho^{n} \alpha}\right|,
$$

which has approximately the value $(\bar{\alpha}-\alpha) z / \rho^{n} \alpha \bar{\alpha}$, and as $n$ becomes negatively infinite we write

$$
\log \left|\frac{z-\rho^{n} \bar{\alpha}}{z-\rho^{n} \alpha}\right|=\log \left|\frac{1-\rho^{n} \bar{\alpha} / z}{1-\rho^{n} \alpha / z}\right|,
$$

which has approximately the value $\rho^{n}(\alpha-\bar{\alpha}) / z$; the convergence of the series in (7) follows. Denote the sum of the series by $V_{1}(z)$, which then is harmonic at every finite point of the plane other than the origin and the points $\rho^{n} \alpha$ and $\rho^{n} \bar{\alpha}$. The function $V_{1}(z)$ is continuous and vanishes on the axis of reals $(z \neq 0)$.

In the formula for $V_{1}(z)$ we replace $z$ by $\rho z$, and we have

$$
\frac{\rho z-\rho^{n} \bar{\alpha}}{\rho z-\rho^{n} \alpha}=\frac{z-\rho^{n-1} \bar{\alpha}}{z-\rho^{n-1} \alpha},
$$

so the two series for $V_{1}(z)$ and $V_{1}(\rho z)$ are identical except for notation: $V_{1}(\rho z) \equiv V_{1}(z)$, and this equation is true for all values of $z$ for which $V_{1}(z)$ is defined. 
The function $V(z)-V_{1}(z)$ is harmonic, when suitably defined in the points $\rho^{n} \alpha$, at every point of $R$, and is continuous and equal to zero on the axis of reals $(z \neq 0)$. From the equation $V(\rho z)-V_{1}(\rho z)$ $\equiv V(z)-V_{1}(z)$ it follows that this difference is bounded in the neighborhoods of the origin and of the point at infinity in $R$, hence vanishes identically in $R$, and equation (7) is established. The essence of Theorem 3 can be summarized in the statement that $V(z)$ is of class $K$, in the region $R_{1}$ consisting of $R$ with the points $\rho^{n} \alpha$ deleted.

Theorem 3 is to be used in proving the following theorem.

THEOREM 4 . Let $R$ be the annular region bounded by disjoint Jordan curves $C_{1}$ and $C_{2}$, and let $\zeta_{1}, \zeta_{2}, \cdots, \zeta_{m}$ be points of $R$. Let the function $U(z)$ be harmonic in $R$ except in the points $\zeta_{k}$, let the sum $U(z)+\lambda_{k} \log \mid z$ $-\zeta_{k} \mid$ with $\lambda_{k}>0$ be harmonic throughout the neighborhood of $\zeta_{k}$ when suitably defined at $\zeta_{k}$, and let $U(z)$ be bounded in the neighborhood of $C_{1}$ and $C_{2}$, continuous and non-negative at all except perhaps a finite number of points of $C_{1}$ and $C_{2}$.

If the function $U(z)$ vanishes at all points of $C_{j}, j=1$ or 2 , then any region $\omega\left(z, C_{j}, R\right)>\mu(\geqq 1 / 2)$ which contains no point $\zeta_{k}$ contains no critical point of $U(z)$.

If the universal covering surface of $R$ is mapped onto the interior of a circle, and if non-euclidean geometry is defined in $R$ by means of the map, then any non-euclidean convex set of $R+C_{1}+C_{2}$ which contains all the $\zeta_{k}$ and contains all points of $C_{1}$ and $C_{2}$ at which $U(z)$ is positive also contains all critical points of $U(z)$.

Theorem 4 is proved by mapping the universal covering surface of $R$ onto the interior $R^{\prime}$ of the unit circle $\Gamma$ in the w-plane. The curves $C_{1}$ and $C_{2}$ each counted infinitely often map into complementary $\operatorname{arcs} \Gamma_{1}$ and $\Gamma_{2}$ of $\Gamma$. Each function $\lambda_{k} g\left(z, \zeta_{k}, R\right)$ vanishes on $C_{1}$ and $C_{2}$, and is transformed into $\lambda_{k}$ times a function of precisely the type considered in Theorem 3; each function of this type is in a suitable subregion of $R^{\prime}$ the limit of a sum of functions of the type which appears as the first term of the second member of (4), with positive coefficients. The function $U(z)-\sum_{k=1}^{m} \lambda_{k} g\left(z, \zeta_{k}, R\right)$ is transformed into a function bounded and harmonic except for removable singularities in $R^{\prime}$, which may have infinitely many discontinuities on $\Gamma$, but which can be represented as the limit of a sum of the type that appears as the last term of (4), with positive coefficients. Each locus $\omega\left(w, \Gamma_{k}, R^{\prime}\right)=\mu$ is the image of the locus $\omega\left(z, C_{k}, R\right)=\mu$ under the conformal map. Theorem 4 is a consequence of our generalization of Theorem 2.

Theorem 4 is the analog of Corollary 1 rather than of Theorem 2. 
If in Theorem 4 we omit the requirements $\lambda_{k}>0$ and $U(z) \geqq 0$ on $C_{1}$ and $C_{2}$, it is true that if $\Delta$ denotes the locus $\omega\left(z, C_{1}, R\right)=1 / 2$, if all points $\zeta_{k}$ for which $\lambda_{k}>0$ which do not lie on $\Delta$ lie between $\Delta$ and $C_{1}$, if $U(z)$ is non-negative on $C_{1}$, if all points $\zeta_{k}$ for which $\lambda_{k}<0$ which do not lie on $\Delta$ lie between $\Delta$ and $C_{2}$, if $U(z)$ is nonpositive on $C_{2}$, and if either $U(z)$ is different from zero on an arc of $C_{1}$ or $C_{2}$ or there exist points $\zeta_{k}$ not on $\Delta$, then no critical points of $U(z)$ lie on $\Delta$ itself. The proof here may be given, after conformal map of the universal covering surface of $R$ onto the interior of the unit circle, following the second proof of Corollary 3.

The last part of Theorem 4 admits of immediate extension to an arbitrary region $R$ bounded by a finite number of mutually disjoint Jordan curves, where non-euclidean geometry is defined in $R$ by mapping the universal covering surface of $R$ onto the interior of a circle. Details are left to the reader.

The location of critical points of a linear combination of Green's functions and harmonic measures with positive coefficients for a multiply connected region (compare equation (6)) can also be studied without the use of conformal mapping and non-euclidean geometry; results are obtained corresponding to and by the use of results on the zeros of the derivative of a rational function due to Bôcher and the present writer. ${ }^{6}$

In connection with the methods we are using, a remark due to Bôcher" is appropriate: "The proofs of the theorems which we have here deduced from mechanical intuition can readily be thrown, without essentially modifying their character, into purely algebraic form. The mechanical problem must nevertheless be regarded as valuable, for it suggests not only the theorems but also the method of proof."

\section{HARVARD UNIVERSITY}

${ }^{6}$ Cf. Bull. Amer. Math. Soc. vol. 52 (1946) pp. 346-347.

7 Proceedings of the American Academy of Arts and Sciences vol. 40 (1904) p. 479. 